\title{
APPonco - Um aplicativo móvel para acesso rápido e seguro à informação sobre o câncer infantojuvenil
}

\author{
Jaciana B. da Silva ${ }^{1}$, Jeferson G. de Freitas ${ }^{1}$, José E. S. Moraes ${ }^{1}$, Johnattan D. F. Viana ${ }^{1}$, \\ Thiago Felippe L. Bandeira ${ }^{1}$, Reinaldo B. Braga ${ }^{1}$, Sandra Emília A. Prazeres ${ }^{2}$, Carina T. de Oliveira ${ }^{1}$ \\ ${ }^{1}$ Laboratório de Redes de Computadores e Sistemas (LAR) / Instituto Federal do Ceará (IFCE) \\ ${ }^{2}$ Associação de Combate ao Câncer Infantojuvenil do Ceará / Associação Peter Pan (APP)
}

\begin{abstract}
The Brazilian National Cancer Institute (INCA) estimates for each year of the 2020/2022 triennium 8,460 new cases of cancer in children and adolescents in the 1-19 age group. As the second cause of death in this age group in most regions of Brazil, childhood cancer is considered a serious public health problem. Fortunately, childhood cancer is a disease with a high probability of cure if diagnosed early and treated in specialized centers. Thus, early diagnosis is an important public health strategy for treatment and is directly related to patient survival. This paper presents APPonco, a mobile application for agile and safe access to information about childhood cancer for healthcare professionals (doctors, nurses, nutritionists, etc.) and the general public (patients, families and caregivers). APPonco was a demand from a reference center in the treatment of childhood cancer in Brazil. The paper details the requirements, architecture, and development process of the application. Since 2020, APPonco is available free for Android and iOS. The software is registered in the Brazilian National Institute of Industrial Property (INPI).
\end{abstract}

\section{KEYWORDS}

Cancer, mHealth, Mobile Application

\section{INTRODUÇÃO}

No Brasil, o câncer é a principal causa de morte por doença em crianças e adolescentes de 1 a 19 anos [4]. De acordo com Instituto Nacional de Câncer (INCA), em 2019 foram registradas 2.554 mortes de crianças e adolescentes pela doença e estima que para cada ano do triênio 2020/2022 sejam diagnosticados 8.460 novos casos [4].

Apesar dos indicadores alarmantes, o câncer infantojuvenil é uma doença com alta probabilidade de cura, se diagnosticado precocemente $\boldsymbol{e}$ tratado em centros especializados [6]. Por outro lado, quando o tratamento do câncer é adiado ou inacessível, há uma menor chance de sobrevivência e maiores custos associados ao tratamento [8]. Por essa razão, é necessário rapidez no diagnóstico, resultante dos treinamentos que profissionais de saúde (médicos, enfermeiros, nutricionistas, agentes comunitários, assistentes sociais, psicólogos, etc.) recebem para que, desde cedo, fiquem atentos aos principais sinais e sintomas relacionados ao câncer. Além disso, também é importante a atenção dos familiares e cuidadores, que estão em contato direto com os pacientes e, por isso, são capazes de identificar sintomas com mais agilidade.

In: XX Workshop de Ferramentas e Aplicações (WFA 2021), Minas Gerais, Brasil. Anais Estendidos do Simpósio Brasileiro de Sistemas Multimídia e Web (WebMedia). Porto Alegre: Sociedade Brasileira de Computação, 2021.

(C) 2021 SBC - Sociedade Brasileira de Computação.

ISSN 2596-1683
O diagnóstico precoce é uma estratégia primordial de saúde pública para tratamento e está diretamente relacionado à sobrevida dos pacientes [8]. Nos países desenvolvidos, a sobrevida geral do câncer infantojuvenil melhorou de $30 \%$ na década de 1960 para mais de $80 \%$ na atualidade [3]. Esta taxa de sobrevivência é significativamente menor na maioria dos países latino-americanos [2]. Por exemplo, segundo dados de 2016 do INCA, a sobrevida por câncer na faixa etária até 19 anos é de apenas $64 \%$ no Brasil [4]. Os baixos índices de cura no país refletem falhas tanto no processo de diagnóstico precoce quanto no tratamento especializado.

Desse modo, dada a importância do diagnóstico precoce na cura do câncer infantojuvenil, como também a carência de sistemas computacionais especializados para esse propósito, o presente artigo apresenta o APPonco, um aplicativo móvel para acesso rápido e seguro à informação sobre o câncer infantojuvenil.

O aplicativo APPonco é mais um resultado da cooperação científica e tecnológica estabelecida desde 2014 entre o Instituto Federal de Educação, Ciência e Tecnologia do Ceará (IFCE) e a Associação de Combate ao Câncer Infantojuvenil do Ceará, conhecida como Associação Peter Pan (APP) ${ }^{1}$, visando elevar o índice de cura do câncer e melhorar a qualidade de vida de crianças e adolescentes com a doença por meio das Tecnologias da Informação e Comunicação (TICs). A APP é uma ONG com 25 anos de existência que atua como um centro de excelência no combate à doença no Ceará. Somente em 2020, a APP realizou 53.500 assistências e, atualmente, atende mensalmente cerca de 1.100 crianças e adolescentes [1].

O APPonco é disponibilizado para dois tipos de público: profissionais de saúde, voltado para a capacitação e atualização de profissionais de saúde na detecção de sinais e sintomas do câncer infantojuvenil; e público-geral, voltado para pacientes, familiares e cuidadores, disponibilizando conteúdos digitais especializados sobre o câncer infantojuvenil e informes a respeito de cuidados na higiene e alimentação do paciente. Desde 2020, o APPonco está disponível de forma gratuita nas plataformas Android e iOS.

\section{MATERIAIS E MÉTODOS}

Nesta seção, são apresentados os materiais e métodos utilizados no desenvolvimento do APPonco. O projeto teve uma duração de 1 ano, contando com uma equipe técnica (IFCE) e uma equipe de saúde (APP).

\subsection{Levantamento de Requisitos}

Os requisitos do APPonco foram discutidos em reuniões de Grupo Focal realizadas com profissionais da APP para delinear as funcionalidades do aplicativo. Desse modo, foram definidos quatro

\footnotetext{
$\overline{{ }^{1} \text { www.app.org.br }}$
} 
perfis de usuários. Os requisitos funcionais foram organizados para cada perfil, como seguem:

(1) Profissional de Saúde (médico, enfermeiro, nutricionista, assistente social, etc.):

- Conteúdo especializado sobre o câncer infantojuvenil;

- Fluxogramas de condutas e exames para cada tipo de câncer;

- Chat online com médico plantonista da APP para tirar dúvidas médicas;

- Agenda de eventos na temática do câncer infantojuvenil;

- Perguntas frequentes (Frequently Asked Questions - FAQ);

- Lista e mapa de Serviços SUS especializados no tratamento do câncer infantojuvenil no Brasil.

(2) Público-Geral (pacientes, familiares e cuidadores):

- Conteúdo especializado sobre o diagnóstico precoce de câncer infantojuvenil;

- Tratamentos e seus efeitos colaterais;

- Cuidados com paciente no contexto da conduta de higiene, nutrição, vacinação e psicologia;

- Perguntas frequentes (FAQ);

- Lista e mapa de Serviços SUS especializados no tratamento do câncer infantojuvenil no Brasil.

(3) Plantonista da APP (médico oncologista pediátrico):

- Gestão do Chat online para tirar dúvidas de profissionais de saúde localizados em todo o Brasil sobre o câncer infantojuvenil;

- Criar/Editar/Excluir conteúdos do aplicativo, tais como Agenda de eventos, Serviços SUS, FAQ, preferências do aplicativo, etc.

(4) Administrador:

- Gerenciar permissões dos usuários;

- Visualizar Dashboard de Indicadores no formato de gráficos e mapas.

Os conteúdos especializados foram coletados de manuais, guias e cartilhas especializadas do INCA, Ministério da Saúde, dentre outros; tendo sido todos validados pela equipe de saúde da APP.

\subsection{Arquitetura}

Em paralelo ao levantamento de requisitos, foi realizado um estudo técnico das tecnologias mais adequadas para atender às demandas do APPonco. A arquitetura utilizada para o desenvolvimento do aplicativo é formada por dois módulos principais.

O primeiro é o Módulo de Apresentação, responsável por toda interação com o usuário final. Esse módulo foi desenvolvido utilizando o framework Flutter, que permite compilar nativamente o código para dispositivos móveis Android e iOS a partir de um único código-fonte.

O segundo é o Módulo de Acesso aos Dados, responsável por toda gestão de usuários, FAQ, artigos e outros conteúdos especializados disponibilizados no aplicativo. Nesse módulo foi utilizado o Firebase por conta de sua infraestrutura robusta, segurança na transferência de dados, mecanismos de autenticação, fácil escalabilidade e maior redução de custos com os recursos utilizados. Além dos serviços do Firebase Database para armazenagem dos dados, foi utilizado o Firebase Storage para armazenar imagens e outros arquivos, e o Firebase Cloud Message para envio de notificações para os usuários.

\subsection{Desenvolvimento}

Após o levantamento de requisitos e a definição da arquitetura, foi iniciado o ciclo de desenvolvimento do aplicativo, que foi construído seguindo a metodologia Scrum [7]. As tarefas foram divididas em três grupos principais: modelagem do banco de dados, desenvolvimento das interfaces de usuário e estruturação do código-fonte.

A modelagem do banco de dados foi realizada de maneira a oferecer um grande potencial de escalabilidade, flexibilidade e adaptabilidade, visto que o aplicativo poderia suportar grandes quantidades de usuários e requisições, além de ganhar novas funcionalidades.

O projeto foi construído com foco na usabilidade do usuário, com interface fluida e intuitiva. Para isso, o desenvolvimento da interface gráfica foi realizado adotando a metodologia de design de experiência do usuário proposta por Jesse James Garrett [5].

Na construção do código-fonte foi utilizado o padrão Model-ViewController (MVC), que permitiu separar, em camadas, as responsabilidades sobre as regras de negócio, facilitando a manutenção e reaproveitamento de código. Toda a aplicação móvel foi projetada para que os usuários tivessem acesso aos conteúdos especializados mesmo sem conexão à Internet. Para isso, o aplicativo fez uso de um banco de dados $S Q L i t e$, que replica todo o conteúdo especializado salvo no Firebase Database.

\subsection{Verificação, Validação e Testes}

As funcionalidades desenvolvidas a cada macro entrega passaram por rigorosos processos de verificação, validação e testes, tanto da equipe técnica do IFCE, quanto da equipe médica da APP. Esses processos foram executados compreendendo as especificações do escopo do projeto, assim garantindo a segurança, consistência e corretude das informações de seus usuários.

\subsection{Implantação}

Desde 2020, o APPonco é disponibilizado gratuitamente para download nas plataformas Play Store ${ }^{2}$ e Apple Store ${ }^{3}$.

\subsection{Licença de Software}

O APPonco é de uso gratuito com licença de software proprietária. O software está registrado no Instituto Nacional da Propriedade Industrial (INPI), processo $\mathrm{n}^{\circ}$ BR512020001684-7.

\section{RESULTADOS E DISCUSSÕES}

Por uma limitação de espaço, nesta seção são apresentadas somente as principais telas e funcionalidades do APPonco com foco na visão do Profissional de Saúde e do Público-Geral.

Vídeos demonstrativos do APPonco: https://drive.google.com/ drive/folders/1APToUxwOPu4-QkiHtWxJV3WezSxjq9Nb?usp=sharing

\subsection{Profissional de Saúde}

Conforme exemplificam as telas da Figura 1(a), o APPonco para o Profissional de Saúde disponibiliza diversos conteúdos especializados sobre o câncer infantojuvenil através de interfaces intuitivas em uma linguagem técnica/médica.

\footnotetext{
${ }^{2}$ https://play.google.com/store/apps/details?id=br.edu.ifce.lar.apponco

${ }^{3}$ https://apps.apple.com/us/app/apponco/id1544540338
} 
Na primeira tela da Figura 1(a), o usuário que instala o APPonco escolhe se deseja acessar o APPonco como profissional de saúde ou público-geral. Se for escolhida a primeira opção, o usuário é direcionado para uma tela de cadastro, devendo comprovar seu vínculo como profissional de saúde (imagem de documento do Conselho). Após a aprovação do cadastro do profissional no APPonco, o usuário pode realizar seu login para ter acesso ao conteúdo do APPonco. Para o público-geral não há necessidade de cadastro/login.

Na sequência, é apresentada a tela de Home do profissional de saúde, que contém diversos cards informativos sobre 11 sinais e sintomas do câncer infantojuvenil, bem como os tipos de câncer (2 tumores hematológicos e 8 tumores oncológicos). A tela de Home também possibilita o acesso às outras funcionalidades do APPonco, conforme previsto no levantamento de requisitos (Seção 2.1).

Em seguida, está ilustrada uma tela informativa de um dos tipos de câncer hematológico, a Leucemia Aguda. Conforme esse exemplo, para todos os tumores é apresentada uma breve descrição da doença, seus principais sintomas e um fluxograma de condutas e exames da doença. A tela seguinte ilustra o fluxograma da Leucemia Aguda.

Em seguida, está exposta a tela com a funcionalidade que permite realizar filtros por sinais e sintomas, permitindo que o profissional possa identificar de forma rápida um tipo de câncer infantojuvenil de acordo com uma combinação de sinais e sintomas.

No mais, é exibida a tela de FAQ, com as perguntas frequentemente realizadas sobre o câncer infantojuvenil, sinais e sintomas e procedimentos médicos. Todo o conteúdo do FAQ foi criado por médicos oncologistas pediátricos da APP. Um filtro é disponibilizado na barra superior para facilitar a busca de FAQs por determinado conteúdo. Os profissionais da APP podem criar/editar/excluir os FAQs via uma interface intuitiva no APPonco.

Dando continuidade, é apresentada a tela de 54 serviços SUS (hospitais e institutos) existentes no Brasil. Ao clicar em um serviço, são apresentadas as informações de endereço, telefone, site, o tipo de serviço oferecido, dentre outras observações. Filtros são disponibilizados na barra superior e no botão ao lado da barra para facilitar a busca do serviço SUS, incluindo filtros por estado e/ou cidade. Conforme ocorre com as FAQs, os profissionais de saúde da APP podem criar/editar/excluir os serviços SUS via aplicativo. Na tela ao lado, os serviços SUS são apresentados no formato de mapa com os indicadores de onde esses serviços estão localizados no Brasil.

A próxima tela ilustra a funcionalidade de Chat, que permite que o usuário profissional de saúde do APPonco converse diretamente com os plantonistas da APP (médicos oncologistas pediátricos) para tirar dúvidas não sanadas pelos conteúdos do aplicativo. Para preservar a confidencialidade do aplicativo, os dados mostrados nessa tela de Chat são todos fictícios.

Por fim, é mostrada a tela de notificações, na qual o usuário pode visualizar eventos no contexto do câncer infantojuvenil (treinamentos, palestras, congressos, etc.), assim como notificações do Chat.

\subsection{Público-Geral}

Como mostram as telas da Figura 1(b), o APPonco disponibiliza para o público-geral diversos conteúdos especializados sobre o câncer infantojuvenil, tudo em uma linguagem popular, uma vez que essa parte do aplicativo é direcionada para os pacientes, familiares e cuidadores. Conforme dito anteriormente, esse tipo de usuário não necessita de autenticação para acessar o conteúdo do aplicativo.

Primeiramente, é mostrada a tela de Home que contém os cards informativos sobre o que é o câncer, os tipos de câncer infantojuvenil, as formas de tratamento, efeitos colaterais e cuidados com o paciente. A tela de Home também possibilita o acesso às funcionalidades de FAQ e serviços SUS. Essas duas funcionalidades não são ilustradas por serem semelhantes às telas do profissional de saúde.

Na sequência, é exposta a tela de um tipo de câncer, a Leucemia, com um breve texto abordando a doença, bem como sinais e sintomas, tudo em uma linguagem coloquial.

Em seguida, são exibidas duas telas referentes às formas de tratamento. A partir da primeira, é possível o usuário visualizar as formas de tratamento. Ao clicar em uma das formas, como a quimioterapia, uma breve descrição da forma de tratamento é apresentada.

Por fim, é apresentado o exemplo de uma tela de cuidados com o paciente na temática de higiene. O conteúdo trata temáticas como cuidados para evitar o aparecimento de infecções, higiene da boca, troca de fralda, dicas para banho no paciente, dentre outros.

\section{CONCLUSÕES}

O aplicativo APPonco foi desenvolvido pelo IFCE e APP visando a melhoria do diagnóstico precoce e do tratamento do câncer infantojuvenil no Brasil e, consequentemente, objetivando um aumento dos índices de cura e na melhoria da qualidade de vida dos pacientes e suas famílias. O aplicativo apresentado funciona como uma ferramenta computacional para acesso rápido e seguro à informação sobre o câncer infantojuvenil, servindo para apoio à tomada de decisão não só de profissionais de saúde no diagnóstico e tratamento do câncer infantojuvenil, mas também de pacientes e suas famílias.

Como trabalhos futuros, pretende-se disponibilizar um chatbot para facilitar a interação do público-geral no APPonco.

\section{AGRADECIMENTOS}

À Confederação Nacional de Instituições de Apoio e Assistência à Criança e ao Adolescente com Câncer (CONIACC) e ao Conselho Nacional de Desenvolvimento Científico e Tecnológico (CNPq) pelo financiamento do projeto.

\section{REFERENCES}

[1] APP. 2021. Associação Peter Pan, Números. Disponível em: https://app.org.br/ historia/. Acesso em: 05 de agosto de 2021.

[2] Claudia Allemani et al. 2018. Global surveillance of trends in cancer survival 200014 (CONCORD-3): analysis of individual records for 37513025 patients diagnosed with one of 18 cancers from 322 population-based registries in 71 countries. The Lancet 391, 10125 (2018), 1023-1075.

[3] Friederike Erdmann et al. 2021. Childhood cancer: Survival, treatment modalities, late effects and improvements over time. Cancer Epidemiology 71 (2021). Childhood Cancer: A Global Perspective.

[4] INCA. 2021. Instituto Nacional do Câncer. Câncer infantojuvenil. Disponível em: https://www.inca.gov.br/tipos-de-cancer/cancer-infantojuvenil. Acesso em: $15 \mathrm{de}$ agosto de 2021.

[5] Garrett Jesse James. 2011. The elements of user experience. Chapter 3 (2011), 42-52.

[6] Paula Carolina Guzman et al. 2021. Childhood cancer in Latin America: from detection to palliative care and survivorship. Cancer Epidemiology 71 (2021). Childhood Cancer: A Global Perspective.

[7] Ken Schwaber and Mike Beedle. 2002. Agile software development with Scrum. Vol. 1. Prentice Hall Upper Saddle River.

[8] WHO. 2017. Guide to Cancer Early Diagnosis. World Health Organization (2017). Disponível em: https://www.who.int/publications/i/item/guide-to-cancer-earlydiagnosis. Acesso em: 06 de agosto de 2021. 

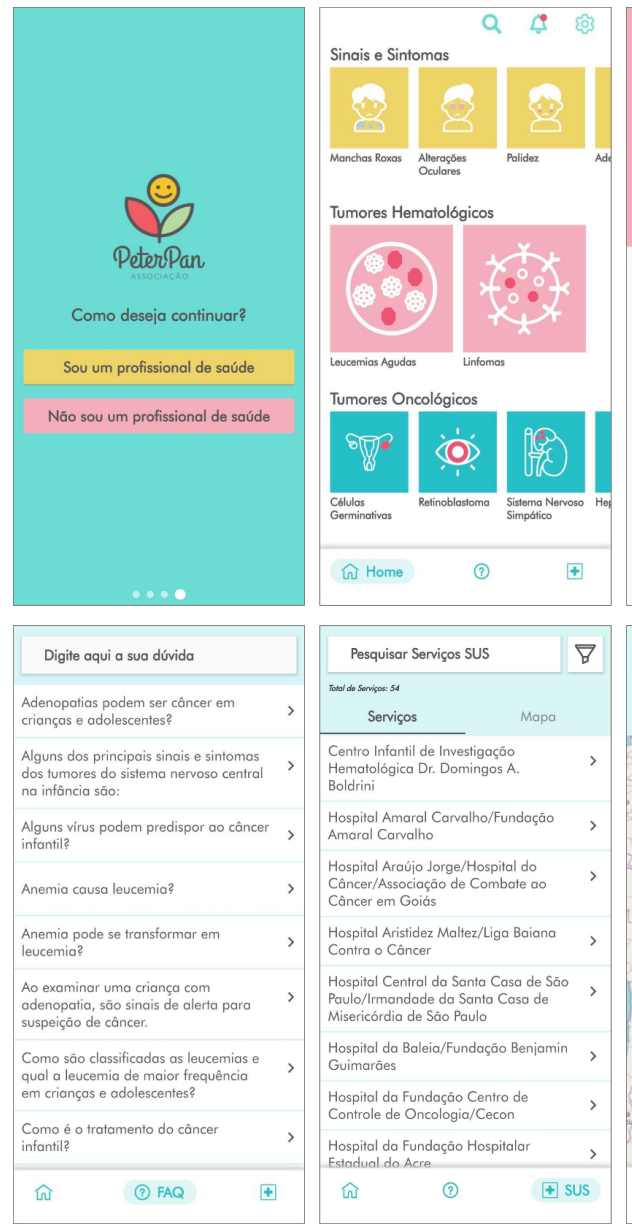

(a) Profissional de Saúde.
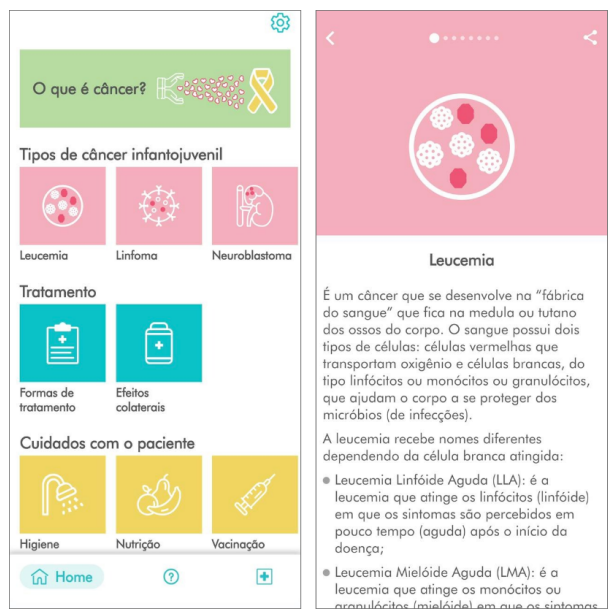
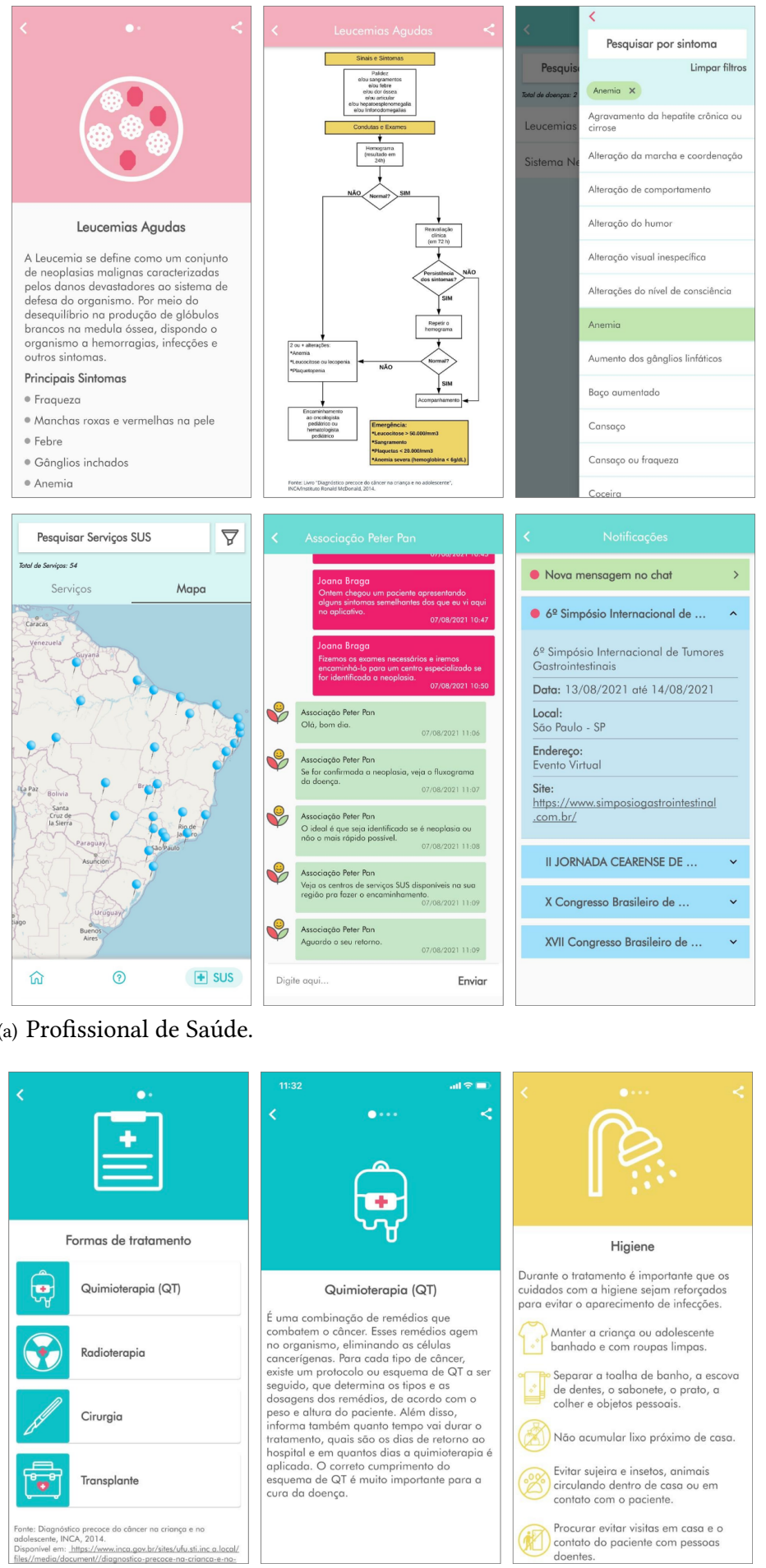

(b) Público-Geral.

Figure 1: Exemplos de telas e funcionalidades do APPonco. 